\section{Exceso de mortalidad durante la pandemia de COVID-19, revisión narrativa}

RODRIGO CERDA THOMAS ${ }^{1, a}$

\section{Excess mortality during the COVID-19 pandemic, a narrative review}

Capturing all the deaths caused by COVID-19 through epidemiologic surveillance based on individual case notification is difficult, therefore, all-cause excess mortality (EM) is an appropriate measure for geographic comparisons and monitoring of the application of non-pharmacological sanitary measures. This is a narrative review of the literature about the observed EM during the COVID-19 pandemic. A research was made on scientific databases (Google Scholar, Pubmed, Virtual Health Library) using the terms "excess mortality", "excess deaths", "COVID-19", "coronavirus", "SARS-CoV-2". It included publications between 2019 and June 16, 2020. Twenty-seven articles were selected from 116 publications found. Most of them correspond to original articles with an analytical ecological study design. They confirm EM in the locations studied, with higher proportion of men and older people and with remarkable spatial heterogeneity. The need to optimize the standardization and updating of mortality registration and reporting mechanisms is highlighted. At a local level, data about EM that will allow these analyses is incipiently becoming available.

(Rev Med Chile 2020; 148: 1647-1651)

Key words: COVID-19; Epidemiological Monitoring; Mortality; Pandemics.
${ }^{1}$ Escuela de Salud Pública,

Universidad de Chile. Santiago,

Chile.

${ }^{a}$ Residente Programa de

formación de especialidad

médica en Salud Pública.

Trabajo no recibió financiamiento

El autor declara no tener

conflictos de interés.

Recibido el 28 de julio de 2020, aceptado el 16 de noviembre de 2020.

Correspondencia a: Rodrigo Cerda Thomas Presidente Balmaceda 2150, Depto. 1607, Santiago. rodrigocerdathomas@gmail.com

\section{A}

inicios de 2020, la Organización Mundial de la Salud reconoció la situación pandémica del nuevo coronavirus (SARSCoV-2), causante de la enfermedad por coronavirus 2019 (COVID-19) ${ }^{1}$. La pandemia ha alterado significativamente la actividad clínica normal, asimismo ha evocado respuestas conductuales y gubernamentales con importantes efectos en la actividad económica ${ }^{2,3}$.

Existe dificultad para capturar toda la mortalidad causada por COVID-19 a través de vigilancia epidemiológica basada en notificación individual de casos, dada la escasez de recursos de laboratorio, sobrecarga de la red de cuidados y el amplio espectro clínico de presentación de la enfermedad. Por esto, el exceso de mortalidad (EM) por cualquier causa es una medida más apropiada para realizar tanto comparaciones geográficas como monitorización de la aplicación de medidas sanitarias no farmacológicas (e. g., cuarentenas) ${ }^{4}$.

Los datos de exceso de mortalidad evitan el conteo inadecuado de muertes atribuidas a subreporte de muertes por COVID-19 y de otras condiciones que no han recibido tratamiento. Por ejemplo, existen reportes de disminución de consultas en servicios de urgencia de enfermedades no relacionadas con COVID-19, lo que podría influir en aumentar la mortalidad ${ }^{5}$.

El propósito de este artículo es realizar una inspección narrativa de la literatura publicada en relación al exceso de mortalidad observado durante la pandemia de COVID-19, con especial énfasis en reconocer la información necesaria para la replicación del estudio a nivel local. 


\section{Materiales y Métodos}

Se realizó una búsqueda en repositorios científicos disponibles en formato electrónico, específicamente Google Scholar, Pubmed y Biblioteca Virtual en Salud. Fueron utilizados los siguientes términos: "excess mortality", "excess deaths", "COVID-19", "coronavirus", "SARS-CoV-2". La búsqueda incluye los hallazgos publicados en 2019 y 2020 (16 de junio de 2020). Las publicaciones encontradas fueron tabuladas, describiendo: año, idioma, especie, tipo de publicación, diseño de estudio, revisión o no por pares, revista de publicación, disponibilidad de resumen.

Se establecieron como criterios de inclusión: artículos referidos a especie humana, en idioma español o inglés, cuyo objetivo fuera el estudio de exceso de mortalidad durante pandemia COVID-19. Se incluyó en los resultados todo tipo de documento. Se excluyeron aquellos textos con referencia a especies no humanas o cuyo objetivo fuera distinto al análisis del exceso de mortalidad (e. g., análisis de letalidad o su asociación con factores de riesgo específicos).

Entre las publicaciones seleccionadas se realizó una síntesis general de la metodología y se extrajeron conclusiones generales.

\section{Resultados}

\section{De la búsqueda bibliográfica}

En total, fueron encontradas 116 publicaciones (29 duplicadas, 88 publicaciones únicas). Al considerar criterios de inclusión y exclusión fueron seleccionadas 27 publicaciones únicas. El uso de términos de búsqueda en español no aportó nuevos hallazgos. Se encuentra disponible la tabulación de los hallazgos de forma abierta. Entre las publicaciones seleccionadas, 23 corresponden a artículos originales, 2 reportes y 2 cartas al editor. Mayoritariamente el diseño de estudio es ecológico analítico. Asimismo, sobre la mitad de los artículos son prepublicaciones, no indexados a revistas con revisión por pares. Veintiuno de los artículos tomaron como referencia un solo país (Italia: 7, Reino Unido: 4, Estados Unidos: 4, Portugal: 2, España: 1, México: 1, Suecia: 1, Brasil: 1) y 3 realizan una comparación internacional.

El exceso de mortalidad se obtiene mediante la diferencia de la mortalidad observada con la mortalidad esperada en un mismo tiempo. La mortalidad en las distintas publicaciones fue analizada tanto en términos absolutos (recuento de defunciones) como relativos (tasa de mortalidad, razón de tasas, riesgo relativo de muerte). Para el establecimiento de la mortalidad esperada fueron identificadas tres metodologías, que grosso modo corresponden a (i) promedio o mediana de valores previos al evento en estudio; (ii) proyección desde el modelamiento de la curva de mortalidad previa y (iii) aplicación de probabilidades (usualmente riesgo relativo de morir) en una distribución de población dada. El establecimiento de la mortalidad observada puede realizarse por (i) recuento de frecuencia absoluta o (ii) por aplicación de probabilidades (riesgo relativo de morir) ante un cambio en la distribución de características de riesgo en una población dada. Sobre todo, en el caso de modelamiento de la mortalidad esperada, se utilizan frecuentemente parámetros suavizadores que permiten capturar los efectos cíclicos y seculares de las tendencias de mortalidad modeladas o que consideran el efecto de otras variables (como contaminación atmosférica).

\section{De las publicaciones seleccionadas}

En la Tabla 1 se muestran los resultados del EM encontrado en cada unidad territorial, con descripción de las semanas cronológicas de 2020 que considera cada publicación. Se informan observaciones de contextualización de los resultados donde se requiera.

\section{Aspectos destacables}

Múltiples publicaciones destacan el aporte diferencial por sexo y grupo de edad al EM. En concreto, serían hombres y personas de edad avanzadas quienes más contribuyen al $\mathrm{EM}^{3,4,14-17,6-13}$. Esto mismo puede verse reflejado en la disminución de la esperanza de vida ${ }^{4,18}$.

Casi la totalidad de los artículos encontrados destacan la importancia de un sistema de vigilancia de la mortalidad continuo, actualizado e idealmente estandarizado, proveyendo de información que permita comparabilidad tanto interna como externa y con el menor desfase posible. Los estudios que realizan un seguimiento temporal más estrecho se basan en plataformas y sistemas electrónicos de registro y estadísticas de defunciones, destacan los sistemas de reporte de defunciones de Portugal, Italia, Reino Unido (Inglaterra y Gales) $y$ del estado de Nueva York (EUA) $)^{2,3,5-8,12,19-21,22-25}$. 
Tabla 1. Resumen de publicaciones y sus principales hallazgos de EM

\begin{tabular}{|c|c|c|c|c|}
\hline Publicación & Territorio & $\begin{array}{c}\text { Período } \\
\text { evaluado* }\end{array}$ & $\begin{array}{l}\text { Exceso de } \\
\text { mortalidad** }\end{array}$ & $\begin{array}{c}\text { Porcentaje atribuido } \\
\text { a COVID-19*** }\end{array}$ \\
\hline Olson et al. ${ }^{5}$ & Estado de Nueva York (estado) & $11-18$ & $\begin{array}{c}24.172 \\
(\text { IC95\% } 22.980-25.364)\end{array}$ & $78 \%$ \\
\hline Rivera et al. ${ }^{6}$ & EUA (país) & $13-16$ & $\begin{array}{c}53.259 \\
\text { (IC95\% 44.256-62.263) }\end{array}$ & $65,2 \%$ \\
\hline Wetzler \& Wetzler ${ }^{7}$ & EUA (país) & $14-18$ & $54.560^{\mathrm{a}}$ & $100 \%$ \\
\hline Friedman et al. ${ }^{8}$ & Tijuana (ciudad, México) & $16-19$ & $\begin{array}{c}195^{\mathrm{b}} \\
(\text { IC95\% 136-254) }\end{array}$ & $4,1 \%$ \\
\hline Freitas et al. ${ }^{9}$ & $\begin{array}{l}\text { Manaus, Fortaleza, Recife, Río de } \\
\text { Janeiro, Sao Paulo, Porto Alegre } \\
\text { (ciudades, Brasil) }\end{array}$ & $11-16$ & 5.344 & $52 \%$ \\
\hline Vandoros S. ${ }^{10}$ & Inglaterra y Gales (naciones) & $10-18$ & $\begin{array}{c}8.708^{c} \\
(I C 95 \% 4.235-13.181)\end{array}$ & $0 \%$ \\
\hline Thomas DM"11 & Inglaterra y Gales (naciones) & $13-21$ & $\begin{array}{c}56769 \\
(\mathrm{DE} 4931)\end{array}$ & $76,7 \%$ \\
\hline $\begin{array}{l}\text { Kontopantelis } \\
\text { et al. }{ }^{12}\end{array}$ & Inglaterra y Gales (naciones) & $11-19$ & $\begin{array}{c}47.243 \\
\text { (IC95\% 46.671-47.815) }\end{array}$ & $78,9 \%$ \\
\hline Vieira et al..$^{13}$ & Portugal (país) & $12-15$ & 1.255 & $49 \%$ \\
\hline Nogueira et al. ${ }^{14}$ & Portugal (país) & $10-17$ & $\begin{array}{c}1.821 \\
\text { (Min-Máx: 192-4.022) }\end{array}$ & $43,1 \%$ \\
\hline $\begin{array}{l}\text { Ochoa Sangrador } \\
\text { et al. } .^{15}\end{array}$ & $\begin{array}{l}\text { Castilla y León (comunidad } \\
\text { autónoma) }\end{array}$ & $10-13$ & $\begin{array}{c}775 \\
(\text { IC95\% 553-1.044) }\end{array}$ & $75,5 \%$ \\
\hline Modi et al. ${ }^{16}$ & Italia (país) & $16-19$ & $\begin{array}{c}51.000 \\
(49.000-53.000)\end{array}$ & $66,7 \%$ \\
\hline Ghislandi et al. ${ }^{17}$ & Italia (país) & $10-16$ & 45.033 & $46,7 \%$ \\
\hline Blangiardo et al. ${ }^{18}$ & Italia (país) & $10-18$ & $\begin{array}{c}41.030 \\
\text { (IC95\% 35.600-46.295) }\end{array}$ & $68,1 \%$ \\
\hline
\end{tabular}

*Semanas cronológicas 2020. **Número de defunciones totales estimadas y rango donde se indique. ***Según el número de defunciones o porcentaje descrito en cada publicación. ${ }^{\mathrm{a} E M}$ estimado por fracción atribuible de COVID-19. ' ${ }^{\mathrm{E}} \mathrm{CM}$ extrahospitalaria. 'EM estimado considerando muertes por causa distinta a COVID-19. EUA: Estados Unidos de América. DE: desviación estándar.

Se presenta también una alta heterogeneidad espacial del EM, describiéndose incluso como granularidad según lo observado, por ejemplo, en el norte de Italia al realizar análisis en distintos niveles territoriales (región, provincia, municipalidad, ciudad). Al utilizar zonas muy extensas en los análisis, puede no capturarse la presencia de EM por esta misma variabilidad territorial, lo mismo ocurre con unidades territoriales muy pequeñas, dado que los márgenes de confiabilidad que otorgan los métodos estadísticos pueden ser demasiado amplios, dificultando la interpretación estadística de los resultados ${ }^{6-11,15,26}$.

Finalmente, una de las hipótesis respecto al aumento de la mortalidad por sobre lo reportado oficialmente debido a COVID-19, es que esta diferencia puede deberse no solo al subreporte de la misma patología, sino también a la disminución de la consulta en los servicios de urgencia. Si bien los resultados y su impacto son objeto de controversia, el fenómeno de disminución de consultas sí queda demostrado en las publicaciones que consideran este punto ${ }^{14,22,24,27}$.

\section{Discusión}

Como conclusión principal, los artículos detectan y documentan EM en los territorios 
analizados, coincidiendo cronológicamente con el período pandémico. Muy ampliamente, se demuestra que las muertes por cualquier causa superan a las detectadas o reportadas como debidas a COVID-19. En este sentido, la metodología de estimación de muertes por cualquier causa cumple con el objetivo de informar sobre la magnitud del efecto en la mortalidad de la emergencia sanitaria.

Se releva la necesidad de contar con sistemas rápidos, eficaces y accesibles para el seguimiento de la mortalidad, que considere períodos cortos de agrupamiento de la información (días, semanas), electrónico y estandarizado. La información obtenida de estas plataformas puede servir como otro factor a considerar para la evaluación y monitorización de medidas públicas de control de la pandemia.

En particular, en Chile, solo recientemente se cuenta con la información que permita replicar algunos de los ensayos mostrados. Siguiendo una metodología simple, como la ocupada por Nogueira y cols. ${ }^{14}$, puede estimarse que el país hasta la semana epidemiológica 25, según el primer reporte de fallecidos entregado por el Departamento de Estadística e Información en Salud (DEIS) en junio de 2020, presenta un EM que puede estimarse en 7.913 muertes (rango: 5.139-10.965 muertes), contrastando con las 4.295 defunciones descritas en el informe epidemiológico del Ministerio de Salud hasta el término de la misma semana epidemiológica ${ }^{28}$.

La mayoría de los estudios provienen de Europa o medios anglosajones, evidenciándose la necesidad de contar con estudios a nivel regional y nacional. La información necesaria para la realización de estos análisis en Latinoamérica no necesariamente cumple con las características mencionadas en forma precedente. El desarrollo de tecnologías que permitan mejorar los estándares de registro y reporte de mortalidad se vuelve una necesidad que debe ser respondida en el corto y mediano plazo.

Agradecimientos: A la Dra. Tania Alfaro Morgado, por su orientación y apoyo en la confección de este artículo.

\section{Referencias}

1. WHO Director-General's opening remarks at the media briefing on COVID-19-11 March 2020 [Internet]. [cited 2020 Jul 26]. Available from: https://www.who. int/dg/speeches/detail/who-director-general-s-opening-remarks-at-the-media-briefing-on-covid-19---11march-2020.

2. Bhatia R. Quantitative Estimation of Covid-19 Related Unemployment On Suicide and Excess Mortality in the United States. medrxiv [Internet]. 2020 May 18 [cited 2020 Jun 1];(1):1-6. Available from: http://medrxiv.org/ cgi/content/short/2020.05.02.20089086.

3. Docherty K, Butt J, de Boer R, Dewan P, Koeber L, Maggioni A, et al. Excess deaths during the Covid-19 pandemic: An international comparison [Internet]. medRxiv. Cold Spring Harbor Laboratory Press; 2020 May [cited 2020 Jun 16]. Available from: http://medrxiv.org/ content/early/2020/04/28/2020.04.21.20073114.abstract

4. Modig K, Ebeling M. Excess mortality from COVID-19. Weekly excess death rates by age and sex for sweden. medRxiv. 2020 May 15;2020.05.10.20096909.

5. Olson DR, Huynh M, Fine A, Baumgartner J, Castro A, Chan HT, et al. Preliminary Estimate of Excess Mortality During the COVID-19 Outbreak - New York City, March 11-May 2, 2020. MMWR Morb Mortal Wkly Rep 2020; 69 (19): 603-5.

6. Aron J, Muellbauer J. Measuring excess mortality: the case of England during the Covid-19 Pandemic. 2020.

7. Blangiardo M, Cameletti M, Pirani M, Corsetti G, Battaglini M, Baio G. Estimating weekly excess mortality at sub-national level in Italy during the COVID-19 pandemic. medRxiv 2020; 2020.06.08.20125211.

8. Freitas ARR, Medeiros NM, Frutuoso L, Beckedorff OA, Martin LMA, Coelho MMM, et al. Use of excess mortality associated with the COVID-19 epidemic as an epidemiological surveillance strategy - preliminary results of the evaluation of six Brazilian capitals. medRxiv 2020; 2020.05.08.20093617.

9. Gibertoni D, Adja KYC, Golinelli D, Reno C, Regazzi L, Fantini MP. Patterns of COVID-19 related excess mortality in the municipalities of Northern Italy. medRxiv. 2020; 2020.05.11.20097964.

10. Kontopantelis E, Mamas MA, Deanfield J, Asaria M, Doran T. Excess mortality in England and Wales during the first wave of the COVID-19 pandemic. medRxiv. 2020; 2020.05.26.20113357.

11. Magnani C, Azzolina D, Gallo E, Ferrante D, Gregori D. How Large Was the Mortality Increase Directly and Indirectly Caused by the COVID-19 Epidemic? An Analysis on All-Causes Mortality Data in Italy. Int J Environ Res Public Health 2020; 17 (10): 3452. Available from: https://www.mdpi.com/1660-4601/17/10/3452 [citado el 16 de junio de 2020].

12. Michelozzi P, de’Donato F, Scortichini M, De Sario M, 
Noccioli F, Rossi P, et al. Mortality impacts of the coronavirus disease (COVID-19) outbreak by sex and age: rapid mortality surveillance system, Italy, 1 February to 18 April 2020. Eurosurveillance 2020; 25 (19): 2000620. Available from: https://www.eurosurveillance.org/content/10.2807/1560-7917.ES.2020.25.19.2000620 [citado el 16 de junio de 2020].

13. Modi C, Boehm V, Ferraro S, Stein G, Seljak U. Total COVID-19 Mortality in Italy: Excess Mortality and Age Dependence through Time-Series Analysis. medRxiv 2020; 1-16. Available from: http://medrxiv.org/content/ early/2020/04/20/2020.04.15.20067074.1.abstract [citado el 1 de junio de 2020].

14. Nogueira PJ, Nobre MDA, Nicola PJ, Furtado C, Vaz Carneiro A. Excess Mortality Estimation During the COVID-19 Pandemic: Preliminary Data from Portugal. Acta Med Port 2020; 33 (6): 376-83.

15. Ochoa Sangrador C, Ramón Garmendia Leiza J, Boillos MJP, Ara FP, Lobato M del PL, de Llano JMA. Impacto de la COVID-19 en la mortalidad de la comunidad autónoma de Castilla y León. Gac Sanit. 2020 May 4.

16. Thomas DM. Excess registered deaths in England and Wales during the COVID-19 pandemic, March 2020 to May 2020. 2020; Available from: http://arxiv.org/ abs/2004.11355 [citado el 16 de junio de 2020].

17. Vieira A, Peixoto Ricoca V, Aguiar P, Abrantes A. Rapid estimation of excess mortality in times of COVID-19 in Portugal - Beyond reported deaths. 2020; Available from: https://doi.org/10.1101/2020.05.14.20100909 [citado el 1 de junio de 2020].

18. Ghislandi S, Muttarak R, Sauerberg M, Scotti B. News from the front: Estimation of excess mortality and life expectancy in the major epicenters of the COVID-19 pandemic in Italy. medRxiv. 2020 May 13;2020.04.29.20084335.

19. Adjiwanou V, Alam N, Alkema L, Asiki G, Bawah A, Béguy $\mathrm{D}$, et al. Measuring excess mortality during the COVID-19 pandemic in low- and lower-middle income countries: the need for mobile phone surveys [Internet]. SocArXiv Papers. 2020. Available from: https://osf.io/ preprints/socarxiv/4bu3q/ [citado el 16 de junio de 2020].

20. Banerjee A, Pasea L, Harris S, Gonzalez-Izquierdo A,
Torralbo A, Shallcross L, et al. Estimating excess 1-year mortality associated with the COVID-19 pandemic according to underlying conditions and age: a population-based cohort study. www.thelancet.com [Internet]. 2020; 395. Available from: www.caliberresearch [citado el 1 de junio de 2020].

21. Felix-Cardoso J, Vasconcelos H, Rodrigues P, Cruz-Correia R. Excess mortality during COVID-19 in five European countries and a critique of mortality analysis data. medRxiv. 2020 May 2;2020.04.28.20083147.

22. Friedman J, Calderon-Villarreal A, Bojorquez I, Vera Hernández C, Schriger D, Tovar Hirashima E, et al. Excess Out-Of-Hospital Mortality and Declining Oxygen Saturation: The Sentinel Role of EMS Data in the COVID-19 Crisis in Tijuana, Mexico. medRxiv. 2020;2020.05.13.20098186.

23. Leon DA, Shkolnikov VM, Smeeth L, Magnus P, Pechholdová M, Jarvis CI. COVID-19: a need for real-time monitoring of weekly excess deaths. Vol. 395, The Lancet. Lancet Publishing Group; 2020. p. e81.

24. Rivera R, Rosenbaum J, Quispe W. Estimating Excess Deaths in the United States Early in the COVID-19 Pandemic [Internet]. medRxiv. Cold Spring Harbor Laboratory Press; 2020 May [cited 2020 Jun 16]. Available from: http://medrxiv.org/lookup/doi/10.1101/2020.05. 04.20090324 .

25. Vandoros S. Excess mortality during the Covid-19 pandemic: Early evidence from England and Wales. Soc Sci Med. 2020; 258: 113101.

26. Piccininni M, Rohmann JL, Foresti L, Lurani C, Kurth T. Use of all cause mortality to quantify the consequences of COVID-19 in Nembro, Lombardy: Descriptive study. BMJ. 2020; 369.

27. Knight A. Letter to the editor about the article "excess Mortality Estimation during the COVID-19 Pandemic: Preliminary Data from Portugal https://doi. org/10.20344/amp.13928." Vol. 33, Acta Medica Portuguesa. CELOM; 2020. p. 446-7.

28. Ministerio de Salud de Chile. Reporte Diario 21 de junio de 2020. Available from: https://cdn.digital. gob.cl/public_files/Campañas/Corona-Virus/Reportes/21.06.2020_Reporte_Covid19vf.pdf [citado el 10 de diciembre de 2020]. 